\title{
Methods of increasing the efficiency of the worm gear
}

\author{
Pavel Melnikov ${ }^{1, *}$ and Svetlana Schegoleva ${ }^{1}$ \\ ${ }^{1}$ Far Eastern Federal University, Campus 10 Ajax Bay, Russky Island, Vladivostok, 690922, Russia
}

\begin{abstract}
This article describes the geometric features of the worm gear, the principle of operation and the theoretical basis for calculating the efficiency. A number of research works aimed at the development of new advanced techniques, both production and use of worm gear were analyzed. Based on the analyzed techniques, one was chosen and confirmed experimentally. Based on the performed researches and experiments, recommendations were developed to improve the efficiency of the worm gear type $2 \mathrm{H}-63$.
\end{abstract}

\section{Introduction}

The use of worm gears is widespread in many areas of industry. Worm gear provides smooth and quiet operation, so it is used in hydraulic, lifting and transport machines, automotive, machine tools, etc.

The main disadvantage of worm gears, affecting the efficiency and benefits of their use, is the relatively low coefficient of performance. A low indicator of this coefficient (approximately 60\%) entails significant energy losses, which makes the use of such reducers unprofitable [1].

The solution to the issue of increasing the efficiency of worm gear is an important and relevant topic. The scientific works of modern scientists prove the relevance of the topic.

In the article by B. Magyar, B. Sauer «Calculation of the efficiency of worm gear drives» presents a physically grounded calculation method to determine the efficiency of worm gear drives. The method proposed by the authors is based on the MEGT tribological simulation, which can determine the local tooth friction coefficients. The authors of the study proved the effectiveness of the proposed method [2].

In a scientific article by E. Mautner, W. Sigmund, J.-P. Stemplinger and K. Stahl «Efficiency of worm gearboxes» conducted experimental studies of large-sized worm gearboxes in order to test knowledge regarding load-carrying capacity and efficiency of their use. The authors of the study during each experiment evaluate the efficiency of the gearbox. The result of this research project is a new technique for processing the components of a worm gear [3].

In scientific works and publications [4-7], special attention is also paid to the issue aimed at eliminating the main drawback - increasing the efficiency of worm gear. One of

\footnotetext{
* Corresponding author: pavel_melnikov98@mail.ru
} 
the main and relevant directions for solving this issue is the development of improved techniques and methods for manufacturing the components of the worm gear.

Thus, this article is devoted to the consideration and study of the geometric features of the worm gear. This is necessary to develop recommendations for existing methods for improving their mechanism and to achieve the most effective work.

\section{Theory}

This article discusses a single-stage worm gear, the basis of which is a single lead (Archimedes) worm and a driven worm wheel. The following is the composition, principle of operation and geometric features of the worm gear.

\subsection{Composition and principle of operation of a single-stage worm gear}

A worm gear (Fig. 1) consists of a leading worm 1 and a driven worm wheel 2, which are located in the protective housing 3. A screw is a screw with a cut thread of a certain type; often the profile of such a thread is close in shape to a trapezoidal thread. The worm wheel has oblique teeth, the profile of which corresponds to the profile of the turns of the worm.

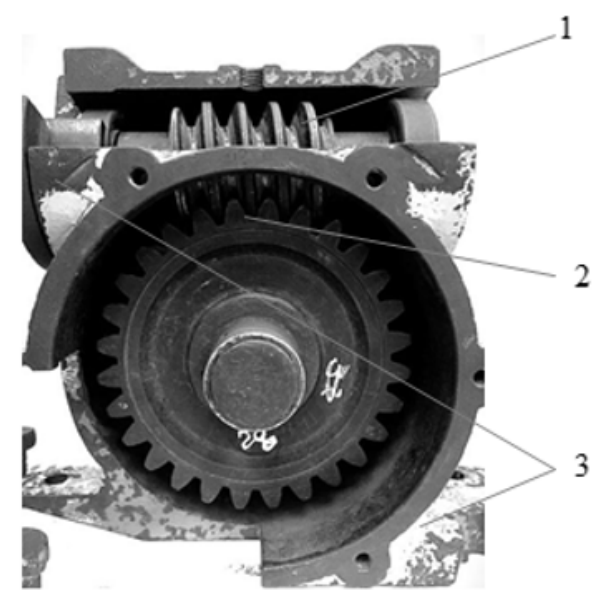

Fig. 1. Single-stage worm gear.

The principle of operation of the worm gear is based on the engagement of the teeth of the worm and the worm wheel. The movement in the transmission is converted on the principle of a helical pair or on the principle of an inclined plane. During the rotation of the worm, its turns move along the axis, due to which the worm wheel (its teeth) move in the same direction [8].

\subsection{The main geometric parameters of the worm and the worm wheel}

The main parameters of the worm gear (Fig. 2a) include: $d_{1}, d_{2}$ - dividing diameters of the worm and the worm wheel; the calculated pitch of the worm $\mathrm{p}$ is equal to the distance between the same side profiles of two adjacent turns of the worm; profile angle $\alpha$ (often equal to $20^{\circ}$ ).

When designing and manufacturing a worm pair, it is necessary to correctly calculate the following geometric parameters: the diameter of the vertices of the turns $d_{a 1}$ and the diameter of the vertices of the hollows of the worm $d_{f 1}$; the diameter of the tops of the teeth 
$d_{a 2}$, and the diameter of the tops of the teeth of the troughs of the teeth of the worm wheel; center distance $a_{w}$, which is equal to half the sum of $d_{1}$ and $d_{2}$ (Fig. 2b).

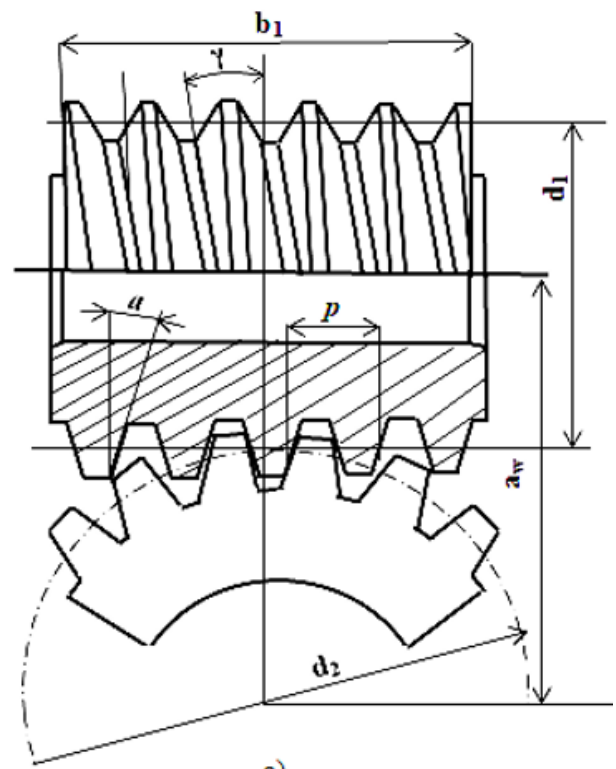

a)

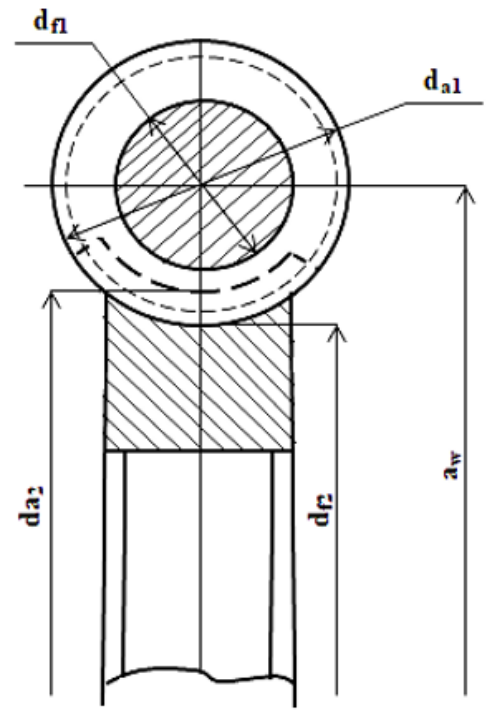

b)

Fig. 2. The main geometric parameters of the worm gear: a) Geometrical parameters of the worm; b) Geometrical parameters of a worm wheel.

In the theory of calculating the parameters of worm gears, an important element is the angle of elevation of the helical line $\gamma$ (Fig. 2a), an acute angle formed between the tangent to the line of the turn of the worm and its section plane. The magnitude of the tangent of the angle of elevation of the helix is the ratio of the turn of the turn $\left(\rho_{z}\right)$ to the length of the initial circumference of the worm:

$$
\operatorname{tg} \gamma=\frac{p_{z}}{\pi d_{1}}=\frac{\pi m z_{1}}{\pi m q}=\frac{z_{1}}{q}
$$

In this formula, $m$ is the engagement modulus, $q$ is the coefficient of the diameter of the worm, $z_{1}$ is the number of entries (turns) of the worm.

There are several types of losses in a worm gear: losses in engagement and bearings, losses due to splashing and moving oil (hydraulic losses). Losses are the main reasons for the low efficiency of the worm gear. The efficiency of the worm gear can be determined by the following formula:

$$
\eta=\eta_{g} \eta_{b} \eta_{\mathrm{h}}
$$

In this formula, $\eta_{g}$ is the gearing efficiency, $\eta_{b}$ is the bearing efficiency, $\eta_{h}$ is the hydraulic loss efficiency.

The main difference between a worm gear and other mechanical gears is the calculation of gearing efficiency.

The efficiency of bearings and hydraulic losses can be calculated by the formulas: 


$$
\eta_{b}=1-\frac{P_{b}}{P_{\mathrm{i}}} \text { and } \eta_{h}=1-\frac{P_{\mathrm{h}}}{P_{\mathrm{i}}}
$$

To use the formula, it is necessary to know the power attributable to friction in bearings $\left(P_{b}\right)$, the power of hydraulic losses $\left(P_{h}\right)$ and the power supplied to the input $\left(P_{i}\right)$.

The calculation of the gearing efficiency (with the leading worm) by the principle of applying formulas (3) is hardly possible, because it is practically impossible to determine the power attributable to the loss in engagement of the worm pair.

Thus, it is necessary to know the helix angle $\gamma$ (formula (1)), and the friction angle $\varphi$. Then, to calculate the gearing efficiency, the formula is used:

$$
\eta_{g}=\frac{\operatorname{tg} \gamma}{\operatorname{tg}(\gamma+\varphi)}
$$

When using formula (4), it is necessary to understand that the meshing efficiency will increase with an increase in the number of approaches (since $\gamma$ increases) and with a decrease in the friction angle $\varphi$.

\section{Analysis of new methods for increasing the efficiency of a worm gear}

In the studied research materials [9-13], the results of studies and experiments are presented. Based on these experiments, author's methods for improving the mechanism of a worm gear and its parts are presented.

The authors of this article have analyzed and experimentally verified the hypothesis proposed in the scientific material «On one of the ways to increase the efficiency of a worm gear», authored by L. Otkidach, B. Baikova. The idea of the hypothesis is to establish a special clip in the area of engagement of the worm and the worm wheel, which creates an isolated area of excess pressure of the oil flow, which comes from the worm in the place of engagement. A special clip is fixed in order to create a stable oil film, which helps to separate the friction surfaces (in the area of engagement of the worm and the worm wheel) and completely eliminate «dry» friction [12].

Studies on the confirmation (refutation) of the hypothesis were carried out on a 2CH-63 type worm gear of the same type with additionally connected elements: a single-phase voltage stabilizer operating in automatic mode, a digital wattmeter, a single-phase electric motor, and connecting couplings. The installation was assembled to lift the load (lifting device), weighing $400 \mathrm{~g}$ to a small height $(30 \mathrm{~cm})$. Instead of a clip, a nozzle was installed on the worm wheel (Fig. 3), made of plastic - a material with a low coefficient of friction $(0,04)$. 


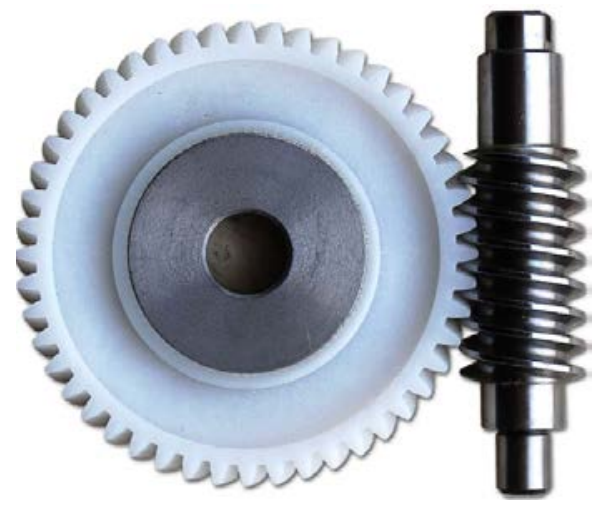

Fig. 3. Worm wheel plastic nozzle

Before starting the experiments, the following tasks were set:

1) To establish the effect of various types of lubricants on the gearing efficiency (friction coefficient) and the overall gearbox efficiency (formula 2);

2) Determine the dependence of power consumption on torque.

The experiments were carried out without changing the mass of the cargo and other components of the installation, only the types of lubricant were changed. TP-30 turbine oil with a low kinetic viscosity of 41.4 to $50.6 \mathrm{~mm}^{2} / \mathrm{s}$ at a temperature of up to $40^{\circ} \mathrm{C}$ was taken as the first version of the lubricant.

The power spent lifting the load was recorded by an automated wattmeter (type TP-Link HS110). As the second lubricant, we used transmission oil - 75W90 at a similar temperature with a higher kinetic viscosity (100-120 mm2 / s). In fig.4 shows graphs of the dependence of power consumption (W) on torque (T) for various lubricant variants.

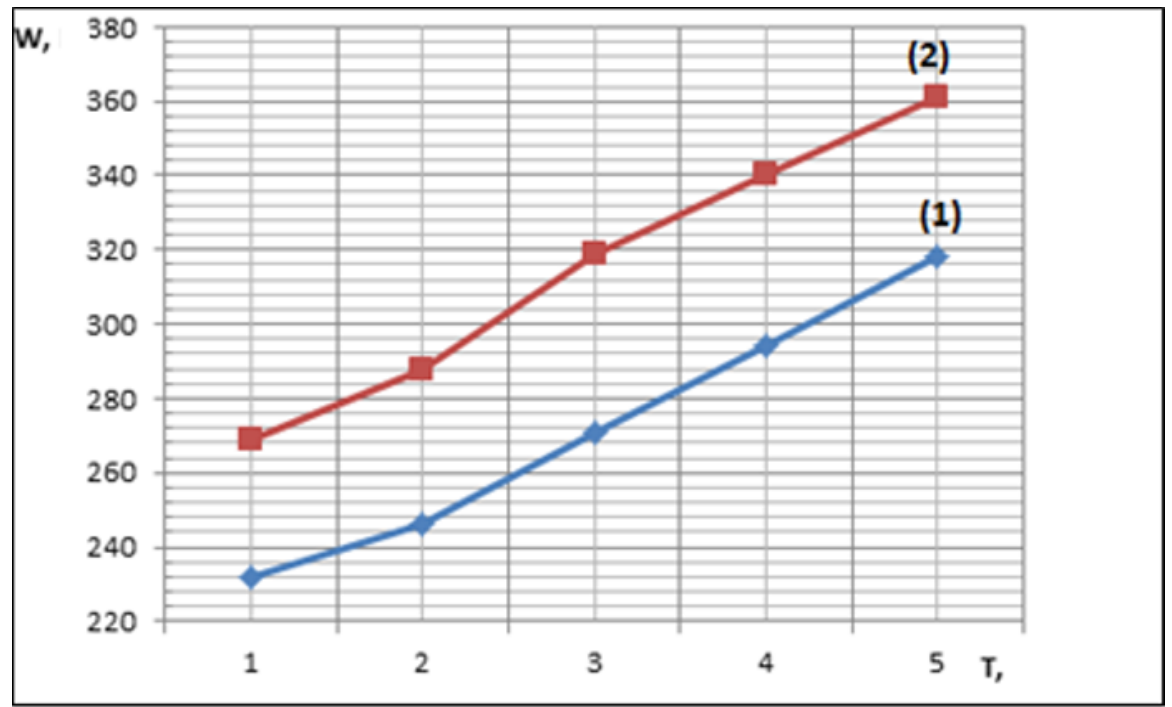

Fig. 4. Graph of power consumption (W) versus torque (T): (1) - TP-30 oil; (2) - 75 W90 oil.

Thus, based on the results obtained (Fig. 4), the following conclusion was formulated: when using transmission oil $75 \mathrm{~W} 90$, the power consumption is approximately $15 \%$ higher than when using TP-30 oil at the same torques. The obtained indicator suggests that the installed special nozzle in the area of engagement of the worm and the worm wheel allows the use of lubricants with a lower viscosity (turbine oil TP-30), while energy costs are 
reduced and the wear of the worm and worm wheel is significantly reduced. Given the errors of the devices, we can say that in both cases the dependence of power consumption on torque is linear.

It was confirmed that this approach to solving the problem allows to reduce the coefficient of friction in the mesh, thereby increasing both the meshing efficiency and the overall efficiency by $4-5 \%$.

From research and observation, suggestions were developed to improve the mechanism of the 2CH-63 worm gear in order to increase its efficiency.

\section{Suggestions for improving the mechanism and operation of the worm gear of the type $2 \mathrm{H}-63$}

Based on the formula (4), we can conclude that by reducing the angle of friction (or coefficient of friction) - the overall efficiency of the gearbox will increase. To solve this issue, in addition to installing a special nozzle, it is proposed to use better types of sealing devices used to connect moving parts (glands) in the production of a worm gear [12]. During the experiments, higher quality seals were used compared to those that were offered in the configuration from the manufacturer. This approach will minimize friction and wear of gear parts.

To increase the efficiency and increase the service life of the 2CH-63 worm gear, it is proposed to increase the width of the worm wheel crown (approximately 0,75 times) and use a harder and more wear-resistant material (for example, bronze alloy) for its manufacture.

The current level of modern innovative engineering technologies allows us to improve the quality of processing of various mechanisms and parts [14]. In particular, it is proposed to pay attention to new technologies that improve the quality of processing of the worm and the crown of the worm wheel (minimizing friction). This approach will improve the pairing of the worm and the worm wheel; reduce the losses attributable to heating the gearbox during its operation, thereby increasing the efficiency.

\section{Suggestions for improving the mechanism and operation of the worm gear of the type $2 \mathrm{H}-63$}

In this paper, the following recommendations are presented that increase the efficiency and wear resistance of worm gear parts:

1) The installation in the area of engagement of the worm and the worm pair of a special nozzle made of a material having a low coefficient of friction will increase the efficiency by $4-5 \%$.

2) To reduce the coefficient of friction (subsequently increase the efficiency), it is recommended to use better seals. The use of high-quality sealing devices will minimize wear of gearbox parts.

3) The use of the hardest and wear-resistant material in the manufacture of the worm and the worm wheel will increase the life of the gearbox. An increase in the width of the worm wheel crown and advanced technologies for processing gearbox parts will lead to a higher efficiency value.

\section{References}

1. Shadsky G.V., Feofiolova I.I. Design and technological features of a worm gear with a cylindrical worm. Izvestiya TulGu, 2017, no.8, pp. 316-322. (In Russian) 
2. Magyar, Balazs \& Sauer, Bernd. (2014). Calculation of the efficiency of worm gear drives. 10.1533/9781782421955.15.

3. Mautner, Eva \& Sigmund, Werner \& Stemplinger, J.-P \& Stahl, Karsten. (2015). Efficiency of worm gearboxes. Proceedings of the Institution of Mechanical Engineers, Part C: Journal of Mechanical Engineering Science. 230. 10.1177/0954406215602286.

4. Nikolaeva E.V., Nikolaev M.Yu., Molodtsov A.S., Pimneva N.E., Kazakov M.V. Measuring helical gears and worm wheels on a coordinate measuring machine. Omsk Scientific Herald, 2018, no.4, pp. 140-145. (In Russian)

5. Gupta K., Jain N. K. On surface integrity of miniature spur gears manufactured by wire electrical discharge machining // The International Journal of Advanced Manufacturing Technology. 2014. № 72(9-12). P. 1735-1745. https://doi.org/10.1007/s00170-0145772-0.

6. B. Magyar, B. Sauer, Calculation of the Efficiency of Worm Gear Drives, Power Transmission Engineering, 9 (4), 2015:52-56.

7. Kim, Seong \& Shin, Min \& Byun, Jung \& O, Kwang \& Chu, Chong. (2012). Efficiency Prediction of Worm Gear with Plastic Worm Wheel. International Journal of Precision Engineering and Manufacturing. 13. 10.1007/s12541-012-0021-7.

8. Dudás, I.. (2004). The Theory and Practice of Worm Gear Drives. The Theory and Practice of Worm Gear Drives. 1-314.

9. Kim, Seong \& Shin, Min \& Byun, Jung \& O, Kwang \& Chu, Chong. (2012). Efficiency Prediction of Worm Gear with Plastic Worm Wheel. International Journal of Precision Engineering and Manufacturing. 13. 10.1007/s12541-012-0021-7.

10. Gupta K., Jain N. K., Laubscher R. F. Spark erosion machining of miniature gears: a critical review //The International Journal of Advanced Manufacturing Technology. 2015. № 80 (9-12). P. 1863-1877. https://doi.org/10.1007/s0017.

11. Tsukanov O. N. Analysis of gearing of worm-helical gears in generalizing parameters. News of higher educational institutions - Engineering, 2017. no. 4, pp. 3-10. (In Russian)

12. Otkidach L.G., Baykov B.A. About one way to increase the efficiency of a worm gear. Engineering Herald, 2015 . no. 10, pp. 26-33. http://engsi.ru/doc/821787.html (In Russian)

13. Shimizu, Y. \& Watanabe, K.. (2009). Investigation into improving efficiency of worm gear with new tooth profile for EPS. 30. 151-158.

14. Wilk-Kołodziejczyk, Dorota \& Opalinski, Andrzej \& Nawarecki, E. \& S., KluskaNawarecka. (2016). Exploration of WEB resources in the domain of metal processing technologies. Metalurgija. 55. 127-130. 